\title{
GROUP CLASSIFICATION OF A CLASS OF SEMILINEAR PSEUDOPARABOLIC EQUATIONS
}

\author{
A.V. PANOV
}

\begin{abstract}
Group classification is implemented for a pseudoparabolic partial differential equation with two parameters. Equivalence transformations groups are found and used for classification of the equation parameters. Kernels of principal symmetries groups are found for the equations. Principal symmetries groups are found for specifications of parameters expanding the kernel of transformations groups. The obtained submodels are summarized in a table at the end of the paper.
\end{abstract}

Keywords: Lie algebra, group classification, submodels programm.

Mathematics Subject Classification: 35B06, 35K58, 35K70

\section{INTRODUCTION}

We consider the semilinear equation

$$
\alpha u_{t}-u_{t x x}=f(u)
$$

of pseudoparabolic type (see the terminology in [1], [2]), where $\alpha$ and $f=f(z)$ are constant and functional parameter, $u=u(t, x)$ is the unknown function. As $f(z)=e^{z}$ or $f(z)=z e^{z^{2}}$, this equation describes physical phenomena in semiconductors counting Debye screening and the sources of free charges [2]. In the case $f(z)=z^{3}$ the equation describes quasi-stationary processes in semiconductors under a stationary distribution of sources free charges current [2], and as $f(z)=z$ one deals with the equation of volume charge stratification in a semiconductor [2. The applications of this equation is not limited by semiconductors only. For instance, as $f(z)=z-a z^{3}$, we obtain a famous Hoff equation describing flanged beam buckling [3].

Following "PODMODELI" program [4, we shall equation (1.1) with parameters "big model". The first step of "PODMODELI" program is the group classification. The aim of group classification is calculating of transformations admitted by the equations for each value of parameters. This group is the kernel of full symmetries groups [5]. The aim is also to find the parameters specifications whose full groups expand the kernel.

In Section 2 we seek the group of equivalence transformations for equation (1.1) by employing the approach suggested in [6, 7]. In Section 3 we seek the specifications and the associated full symmetries groups for the equation expanding the kernel of full transformation groups. At that, the form of specifications is reduced to the simplest possible by equivalence transformations found in the second section.

As a result of group classification we find the kernels of full symmetries groups; for $\alpha \neq 0$ the kernel consists of shifts w.r.t. independent variables, as $\alpha=0$ the shifts are completed by the dilatation generated by $X_{3}=x \partial_{x}-2 t \partial_{t}$. We find all the parameters specifications reducing additional equation symmetries. All nonlinear specifications expanding the kernel are in the equivalence classes of functions $e^{u}, u^{\beta}, u^{-3}, e^{u} \pm 1$. As $\alpha \neq 0$, the biggest symmetries algebra

A.V. Panov, Group classification of a Class of Semilinear pSeudoparabolic equations.

(C) PANOV A.V. 2013.

Submitted October 4, 2013. 
corresponds to the equation with the function $f=u^{-3}$, its Lie algebra is five-dimensional. The obtatined submodels are summarized in the table in the end of the work.

\section{EQUiVALENCE TRANSFORMATIONS GROUP}

While implementing group classification, it is important to know which transformations change the parameters in the equation keeping at the same time the differential structure of the equation. These transformations determine equivalence transformations on the set of the parameters of the equation. The symmetries groups of two differential equations corresponding to a pair of different but equivalent parameters are isomorphic.

We write the original equation as

$$
\alpha u_{t}-u_{t x x}-f=0,
$$

assuming that $\alpha, f$ are additional variables independent of independent variables $t, x, u$. We shall seek the generators of continuous equivalence transformations groups as

$$
Y=\tau \frac{\partial}{\partial t}+\xi \frac{\partial}{\partial x}+\eta \frac{\partial}{\partial u}+\mu \frac{\partial}{\partial f}+\nu \frac{\partial}{\partial \alpha},
$$

where the functions $\tau, \xi, \eta, \mu, \nu$ depend on $t, x, u, f, \alpha$ (cf. [6, 7, 8]). We supplement equation (2.1) with equations

$$
\begin{gathered}
f_{t}=0, \quad f_{x}=0, \\
\alpha_{t}=0, \quad \alpha_{x}=0, \quad \alpha_{u}=0,
\end{gathered}
$$

meaning that in the original formulation of the problem $f$ depends on $u$ only, while $\alpha$ is a constant.

We consider system (2.1)-(2.3) as a manifold $\mathfrak{N}$ in the expanded space of corresponding variables. In order to find the admitted groups for the manifold $\mathfrak{N}$, we employ the infinitesimal criterion [5] applying the expanded operator

$$
\widetilde{Y}=Y+\varphi^{t} \frac{\partial}{\partial u_{t}}+\varphi^{t x x} \frac{\partial}{\partial u_{t x x}}+\mu^{t} \frac{\partial}{\partial f_{t}}+\mu^{x} \frac{\partial}{\partial f_{x}}+\mu^{u} \frac{\partial}{\partial f_{u}}+\nu^{t} \frac{\partial}{\partial \alpha_{t}}+\nu^{x} \frac{\partial}{\partial \alpha_{x}}+\nu^{u} \frac{\partial}{\partial \alpha_{u}}
$$

to equations (2.1)-(2.3). We then restrict the result on manifold $\mathfrak{N}$ and obtain the determining equations

$$
\begin{gathered}
\nu u_{t}+\alpha \varphi^{t}-\varphi^{t x x}-\left.\mu\right|_{\mathfrak{N}}=0, \\
\left.\mu^{t}\right|_{\mathfrak{N}}=0,\left.\quad \mu^{x}\right|_{\mathfrak{N}}=0, \\
\left.\nu^{t}\right|_{\mathfrak{N}}=0,\left.\quad \nu^{x}\right|_{\mathfrak{N}}=0,\left.\quad \nu^{u}\right|_{\mathfrak{N}}=0 .
\end{gathered}
$$

The coefficients of operator $\widetilde{Y}$ can be calculated by the expansion formulae involving the differentiation operators

$$
\begin{aligned}
& D_{t}=\frac{\partial}{\partial t}+u_{t} \frac{\partial}{\partial u}+\left(f_{t}+f_{u} u_{t}\right) \frac{\partial}{\partial f}+\left(\alpha_{t}+\alpha_{u} u_{t}\right) \frac{\partial}{\partial \alpha}+u_{t t} \frac{\partial}{\partial u_{t}}+u_{t x} \frac{\partial}{\partial u_{x}}+\ldots, \\
& D_{x}=\frac{\partial}{\partial x}+u_{x} \frac{\partial}{\partial u}+\left(f_{x}+f_{u} u_{x}\right) \frac{\partial}{\partial f}+\left(\alpha_{x}+\alpha_{u} u_{x}\right) \frac{\partial}{\partial \alpha}+u_{x x} \frac{\partial}{\partial u_{x}}+u_{t x} \frac{\partial}{\partial u_{t}}+\ldots, \\
& \widetilde{D}_{t}=\frac{\partial}{\partial t}+f_{t} \frac{\partial}{\partial f}+\alpha_{t} \frac{\partial}{\partial \alpha}+\ldots, \widetilde{D}_{x}=\frac{\partial}{\partial x}+f_{x} \frac{\partial}{\partial f}+\alpha_{x} \frac{\partial}{\partial \alpha}+\ldots, \\
& \widetilde{D}_{u}=\frac{\partial}{\partial u}+f_{u} \frac{\partial}{\partial f}+\alpha_{u} \frac{\partial}{\partial \alpha}+\ldots
\end{aligned}
$$


In view of equations $(2.2),(2.3)$, the operators become

$$
\begin{aligned}
& D_{t}=\frac{\partial}{\partial t}+u_{t} \frac{\partial}{\partial u}+f_{u} u_{t} \frac{\partial}{\partial f}+u_{t t} \frac{\partial}{\partial u_{t}}+u_{t x} \frac{\partial}{\partial u_{x}}+f_{u u} u_{t} \frac{\partial}{\partial f_{u}}+u_{t t t} \frac{\partial}{\partial u_{t t}}+u_{t t x} \frac{\partial}{\partial u_{t x}}+\ldots, \\
& D_{x}=\frac{\partial}{\partial x}+u_{x} \frac{\partial}{\partial u}+f_{u} u_{x} \frac{\partial}{\partial f}+u_{x x} \frac{\partial}{\partial u_{x}}+u_{x t} \frac{\partial}{\partial u_{t}}+f_{u u} u_{x} \frac{\partial}{\partial f_{u}}+\ldots \\
& \widetilde{D}_{t}=\frac{\partial}{\partial t}, \widetilde{D}_{x}=\frac{\partial}{\partial x}, \widetilde{D}_{u}=\frac{\partial}{\partial u}+f_{u} \frac{\partial}{\partial f}+\ldots
\end{aligned}
$$

In accordance with the expansion formulae

$$
\begin{array}{ll}
\varphi^{t}=D_{t}(\eta)-u_{t} D_{t}(\tau)-u_{x} D_{t}(\xi), & \varphi^{x}=D_{x}(\eta)-u_{t} D_{x}(\tau)-u_{x} D_{x}(\xi), \\
\varphi^{x x}=D_{x}\left(\varphi^{x}\right)-u_{t x} D_{x}(\tau)-u_{x x} D_{x}(\xi), & \varphi^{t x x}=D_{t}\left(\varphi^{x x}\right)-u_{t x x} D_{t}(\tau)-u_{x x x} D_{t}(\xi),
\end{array}
$$

and thus

$$
\begin{aligned}
\varphi^{t} & =\eta_{t}+u_{t} \eta_{u}+u_{t} f_{u} \eta_{f}-u_{t} \tau_{t}-u_{t}^{2} \tau_{u}-u_{t}^{2} f_{u} \tau_{f}-u_{x} \xi_{t}-u_{t} u_{x} \xi_{u}-u_{t} u_{x} f_{u} \xi_{f}, \\
\varphi^{x} & =\eta_{x}+u_{x} \eta_{u}+u_{x} f_{u} \eta_{f}-u_{t} \tau_{x}-u_{t} u_{x} \tau_{u}-u_{t} u_{x} f_{u} \tau_{f}-u_{x} \xi_{x}-u_{x}^{2} \xi_{u}-u_{x}^{2} f_{u} \xi_{f} .
\end{aligned}
$$

We first write out equations (2.5), (2.6) to shorten the calculations of coefficients $\varphi^{x x}, \varphi^{t x x}$. We obtain

$$
\begin{aligned}
\left.\mu^{t}\right|_{\mathfrak{N}} & =\left.\left(\widetilde{D}_{t}(\mu)-f_{t} \widetilde{D}_{t}(\tau)-f_{x} \widetilde{D}_{t}(\xi)-f_{u} \widetilde{D}_{t}(\eta)\right)\right|_{\mathfrak{N}}=\mu_{t}-f_{u} \eta_{t}=0, \\
\left.\mu^{x}\right|_{\mathfrak{N}} & =\left.\left(\widetilde{D}_{x}(\mu)-f_{t} \widetilde{D}_{x}(\tau)-f_{x} \widetilde{D}_{x}(\xi)-f_{u} \widetilde{D}_{x}(\eta)\right)\right|_{\mathfrak{N}}=\mu_{x}-f_{u} \eta_{x}=0, \\
\left.\nu^{t}\right|_{\mathfrak{N}} & =\left.\left(\widetilde{D}_{t}(\nu)-\alpha_{t} \widetilde{D}_{t}(\tau)-\alpha_{x} \widetilde{D}_{t}(\xi)-\alpha_{u} \widetilde{D}_{t}(\eta)\right)\right|_{\mathfrak{N}}=\nu_{t}=0, \\
\left.\nu^{x}\right|_{\mathfrak{N}} & =\left.\left(\widetilde{D}_{x}(\nu)-\alpha_{t} \widetilde{D}_{x}(\tau)-\alpha_{x} \widetilde{D}_{x}(\xi)-\alpha_{u} \widetilde{D}_{x}(\eta)\right)\right|_{\mathfrak{N}}=\nu_{x}=0, \\
\left.\nu^{u}\right|_{\mathfrak{N}} & =\nu_{u}+f_{u} \nu_{f}=0 .
\end{aligned}
$$

By the arbitrariness of $f_{u}$ these equations imply that $\eta_{t}=\eta_{x}=0, \mu_{t}=\mu_{x}=0, \nu_{t}=\nu_{x}=\nu_{u}=$ $\nu_{f}=0$. This is why $\eta=\eta(u, \alpha, f), \mu=\mu(u, \alpha, f), \nu=\nu(\alpha)$.

We split equation (2.4) w.r.t. the differential variables. The coefficient at $f_{\text {uuu }}$ is $u_{x}^{2} u_{t} \eta_{f}-$ $u_{t}^{2} u_{x}^{2} \tau_{f}-u_{x}^{3} u_{t} \xi_{f}$. Equating it to zero, we obtain the identities $\eta_{f}=\xi_{f}=\tau_{f}=0$. Making the same for the coefficients at $f_{u u}$, we obtain $\xi_{x u}=0$. We differentiate equation (2.4) by $f$ and taking into consideration $\nu_{f}=\xi_{f}=\tau_{f}=\eta_{f}=0$, we arrive at the equation $\eta_{u}-2 u_{t} \tau_{u}-2 \xi_{x}-3 u_{x} \xi_{u}-\tau_{t}-\mu_{f}=$ 0 . Then $\xi_{u}=\tau_{u}=0$ и $\mu_{f}=\eta_{u}-2 \xi_{x}-\tau_{t}$. Equating to zero the coefficients at various differential variables, we obtain the identities

$$
\begin{aligned}
& u_{t t x}: \tau_{x}=0, \\
& u_{x x x}: \xi_{t}=0, \\
& u_{x x}: 2 \xi_{x t}-u_{t} \eta_{u u}=0 .
\end{aligned}
$$

Thus, $\tau=\tau(t, \alpha), \xi=\xi(x, \alpha), \eta_{u u}=0, \eta=\eta(u, \alpha)$. We rewrite now equation (2.4):

$$
\nu u_{t}+u_{t x} \xi_{x x}+f \eta_{u}+2 \alpha u_{t} \xi_{x}-2 f \xi_{x}-f \tau_{t}-\mu=0 .
$$

We see that $\xi_{x x}=0, \nu+2 \alpha \xi_{x}=0, f \eta_{u}-2 f \xi_{x}-f \tau_{t}-\mu=0$. If we differentiate the latter identity by $t$ and take into consideration that $\mu_{t}=\xi_{t}=\eta_{t}=0$, we obtain $\tau_{t t}=0$. Thus, the solution to the system of the determining equations are the functions $\tau=c_{1}(\alpha)+c_{2}(\alpha) t$, $\xi=c_{3}(\alpha)+c_{4}(\alpha) x, \eta=c_{5}(\alpha)+c_{6}(\alpha) u, \mu=f\left(c_{6}(\alpha)-c_{2}(\alpha)-2 c_{4}(\alpha)\right), \nu=-2 \alpha c_{4}(\alpha)$. Here $c_{i}(\alpha)$ are arbitrary functions of $\alpha$. The basis operator can be chosen as follows,

$$
\begin{aligned}
& X_{1}=c_{1}(\alpha) \partial_{t}, X_{2}=c_{3}(\alpha) \partial_{x}, X_{3}=c_{5}(\alpha) \partial_{u} \\
& X_{4}=c_{2}(\alpha) t \partial_{t}-c_{2}(\alpha) f \partial_{f}, X_{5}=c_{6}(\alpha) u \partial_{u}+c_{6}(\alpha) f \partial_{f}, \\
& X_{6}=c_{4}(\alpha) x \partial_{x}-2 c_{4}(\alpha) f \partial_{f}-2 c_{4}(\alpha) \alpha \partial_{\alpha} .
\end{aligned}
$$


For instance, as $c_{4}=1$, by operator $X_{6}$ we can transform constant $\alpha$ to any other of the same sign. We get three cases $\alpha=1, \alpha=-1, \alpha=0$. Then we do not use operator $X_{6}$ any more in order to keep $\alpha$. Then other equivalence transformation operators form a five-dimensional Lie algebra with the basis

$$
X_{1}=\partial_{t}, \quad X_{2}=\partial_{x}, \quad X_{3}=\partial_{u}, \quad X_{4}=t \partial_{t}-f \partial_{f}, \quad X_{5}=u \partial_{u}+f \partial_{f} .
$$

We consider the action of the projections of these operators on space $\mathbb{R}^{2}(u, f)$. Operator $X_{3}$ gives a shift of variable $u$. The projection $\operatorname{pr}_{(u, f)}\left(-X_{4}\right)=f \partial_{f}$ gives the dilatation in $f$. The dilatation w.r.t. $u$ we obtain as the projectino $\operatorname{pr}_{(u, f)}\left(X_{4}+X_{5}\right)=u \partial_{u}$. Immediately from equation we see two discrete symmetries $\bar{t}=-t, \bar{f}=-f$ and $\bar{u}=-u, \bar{f}=-f$ which produce the reflections in variables $u$ and $f$.

\section{PARAMETERS SPECIFICATIONS}

Let us find the specifications of parameters $\alpha, f$ under which additional symmetries of the equation

$$
\alpha u_{t}-u_{t x x}=f(u)
$$

appear.

We seek the generators of continuous transformations group as

where functions $\tau, \xi, \eta$ depend on $t, x, u$.

$$
Y=\tau \frac{\partial}{\partial t}+\xi \frac{\partial}{\partial x}+\eta \frac{\partial}{\partial u}
$$

We act by the expanded operator

$$
\tilde{Y}=Y+\varphi^{t} \frac{\partial}{\partial u_{t}}+\varphi^{t x x} \frac{\partial}{\partial u_{t x x}}
$$

to the function $F\left(t, x, u, u_{t}, u_{t x x}\right)=\alpha u_{t}-u_{t x x}-f(u)$ describing system (3.1) as $F=0$. By the invariance criterion we obtain

$$
\left.\left(\alpha \varphi^{t}-\varphi^{t x x}-f^{\prime}(u) \eta\right)\right|_{F=0}=0 .
$$

By the operators of total differentiation

$$
D_{t}=\frac{\partial}{\partial t}+u_{t} \frac{\partial}{\partial u}+u_{t t} \frac{\partial}{\partial u_{t}}+u_{t x} \frac{\partial}{\partial u_{x}}+\ldots, \quad D_{x}=\frac{\partial}{\partial x}+u_{x} \frac{\partial}{\partial u}+u_{x x} \frac{\partial}{\partial u_{x}}+u_{x t} \frac{\partial}{\partial u_{t}}+\ldots
$$

and in accordance with the expansion formulae

$$
\begin{array}{ll}
\varphi^{t}=D_{t}(\eta)-u_{t} D_{t}(\tau)-u_{x} D_{t}(\xi), & \varphi^{x}=D_{x}(\eta)-u_{t} D_{x}(\tau)-u_{x} D_{x}(\xi), \\
\varphi^{x x}=D_{x}\left(\varphi^{x}\right)-u_{t x} D_{x}(\tau)-u_{x x} D_{x}(\xi), & \varphi^{t x x}=D_{t}\left(\varphi^{x x}\right)-u_{t x x} D_{t}(\tau)-u_{x x x} D_{t}(\xi)
\end{array}
$$

we write out equation (3.2). We equate the coefficients at the third derivatives to zero

$$
\begin{aligned}
& u_{t t x}: \tau_{x}+u_{x} \tau_{u}=0, \\
& u_{x x x}: \xi_{t}+u_{t} \xi_{u}=0 .
\end{aligned}
$$

In the same way we obtain equations for the coefficients at the second derivatives

$$
\begin{aligned}
& u_{t x}:-2 \eta_{u x}+\xi_{x x}-2 u_{x} \eta_{u u}=0, \\
& u_{x x}:-\eta_{t u}-u_{t} \eta_{u u}=0 .
\end{aligned}
$$

Then $\eta_{u u}=0, \eta_{t u}=0, \xi_{x x}=2 \eta_{x u}$. We split the remaining equation by variable $u_{t}$ to arrive to the identities

$$
\begin{gathered}
-\eta_{x x u}+2 \alpha \xi_{x}=0 \\
\alpha \eta_{t}-\eta_{t x x}+f(u) \eta_{u}-2 f(u) \xi^{\prime}(x)-f(u) \tau^{\prime}(t)-f^{\prime}(u) \eta=0 .
\end{gathered}
$$

It implies easily the following statements. 
Theorem 1. (i) The basis of the kernel of full Lie algebras for the equation $-u_{t x x}=f(u)$ consists of the operators $X_{1}=\partial_{t}, X_{2}=\partial_{x}, X_{3}=2 t \partial_{t}-x \partial_{x}$.

(ii) The basis of the kernel of full Lie algebras for equation (3.1) in the case $\alpha \neq 0$ consists of the operators $X_{1}=\partial_{t}, X_{2}=\partial_{x}$.

The further calculation of the specifications splits into three cases: $\alpha=0, \alpha>0, \alpha<0$. The classification results are written in Table 1 . In what follows, the number of the specification is the number of the column and the row in the table.

The first case is $\alpha=0$. Since $\eta_{u u}=0, \eta_{t u}=0, \eta_{x x u}=0$, we have $\eta=\left(c_{1} x+c_{2}\right) u+b(t, x)$. It follows from $\xi_{x x}=2 \eta_{x u}$ that $\xi=c_{1} x^{2}+c_{3} x+c_{4}$. We substitute these expressions into equation (3.3) to obtain

$$
-b_{t x x}(t, x)+f(u)\left(c_{1} x+c_{2}-4 c_{1} x-2 c_{3}-\tau^{\prime}(t)\right)-f^{\prime}(u)\left(\left(c_{1} x+c_{2}\right) u+b(t, x)\right)=0 .
$$

The case $f^{\prime}(u)=0$ splits into other two.

1.1. If $f=0$, we obtain the equation $b_{t x x}=0$ giving the solution to the determining system of equations

$$
\tau=\tau(t), \xi=c_{1} x^{2}+c_{3} x+c_{4}, \eta=\left(c_{1} x+c_{2}\right) u+c(t) x+d(t)+e(x) .
$$

An infinite-dimensional Lie algebra is associated with this case.

1.2. if $f=$ const $\neq 0$, by the dilatation in variable $f$ we can obtain $f=1$. Then the solution is

$$
\begin{aligned}
& \tau=\tau(t), \xi=c_{1} x^{2}+c_{3} x+c_{4}, \\
& \eta=\left(c_{1} x+c_{2}\right) u+\left(\frac{c_{2}}{2}-c_{3}\right) x^{2} t-\frac{c_{1}}{2} x^{3} t-\tau(t) \frac{x^{2}}{2}+c(t) x+d(t)+e(x) .
\end{aligned}
$$

As $f^{\prime}(u) \neq 0$, we consider two different cases.

1.3. Let $f^{\prime \prime}(u)=0$, then $f=\sigma u+\delta, \sigma \neq 0$. Applying the shift and dilatation in $u$, we can transform the specification to $f=u$. After the splitting of equation (3.4) w.r.t. variables $x, u$ we obtain the identities

$$
\begin{gathered}
c_{1}=0, \tau=-2 c_{3} t+c_{5}, \\
b_{t x x}(t, x)+b(t, x)=0 .
\end{gathered}
$$

Thus, in this case the coefficients of the generators of symmetries groups read as

$$
\tau=-2 c_{3} t+c_{5}, \quad \xi=c_{3} x+c_{4}, \quad \eta=c_{2} u+b(t, x),
$$

where $b(t, x)$ is a solution to equation (3.5).

Suppose $f^{\prime \prime}(u) \neq 0$. Then, differentiating equation (3.4) by $u$, we obtain

$$
f^{\prime}(u)\left(4 c_{1} x+2 c_{3}+\tau^{\prime}(t)\right)+f^{\prime \prime}(u)\left(\left(c_{1} x+c_{2}\right) u+b(t, x)\right)=0 .
$$

Differentiating the latter equation w.r.t. $t$ and $x$ gives the identiy $b_{t x}(t, x)=0$ and thus $b(t, x)=$ $b_{1}(t)+b_{2}(x)$. We differentiate (3.6) w.r.t. $t$ and $x$ separately:

$$
\begin{gathered}
f^{\prime}(u) \tau^{\prime \prime}(t)+f^{\prime \prime}(u) b_{1}^{\prime}(t)=0, \\
4 c_{1} f^{\prime}(u)+c_{1} u f^{\prime \prime}(u)+b_{2}^{\prime}(x) f^{\prime \prime}(u)=0 .
\end{gathered}
$$

In view of equation (3.7) two cases are possible: $\tau=c_{5} t+c_{6}, b_{1}$ is a constant; or $b_{1}^{\prime}(t) \neq 0$, $\tau^{\prime \prime}(t) \neq 0$. In the second case, employing the dilatation and, if needed, the reflection by $u$, we obtain the equation

$$
\frac{f^{\prime \prime}(u)}{f^{\prime}(u)}=-\frac{\tau^{\prime \prime}(t)}{b_{1}^{\prime}(t)}=1 .
$$


Hence, $b_{1}(t)=-\tau^{\prime}(t)+c_{7}, f=\sigma e^{u}+\omega$ or $f=e^{u}+\omega$ after the dilatation in $f$. Now equation (3.8) yields that $c_{1}=0$ and $b_{2}(x)=c_{8}$ is a constant. We re-denote $c_{7}+c_{8}$ by $c_{7}$ and substitute the obtained formulae into (3.4):

$$
\left(e^{u}+\omega\right)\left(c_{2}-2 c_{3}-\tau^{\prime}(t)\right)-e^{u}\left(c_{2} u-\tau^{\prime}(t)+c_{7}\right)=0 .
$$

The case $\omega \neq 0$ does not lead us to the expansion of the kernel of the algebras. If $\omega=0$, then

$$
\tau=\tau(t), \quad \tau^{\prime \prime}(t) \neq 0, \quad \xi=c_{3} x+c_{4}, \quad \eta=-\left(\tau^{\prime}(t)+2 c_{3}\right) .
$$

We return back to the case $\tau=c_{5} t+c_{6}, b=b_{1}+b_{2}(x)$. Consider equation (3.8). Let $c_{1}=0$, then $b_{2}^{\prime}(x)=0$ and equation (3.4) casts into the form

$$
f(u)\left(c_{2}-2 c_{3}-c_{5}\right)-f^{\prime}(u)\left(c_{2} u+b\right)=0,
$$

where $b$ is a constant. Here two cases are possible.

1.4. Let $c_{2}=0$, then condition $b \neq 0$ is necessary for expansion of kernel of Lie algebra. By the dilatation and reflection w.r.t. $u$ equation (3.9) can be reduced to $f^{\prime}(u)=f(u)$. In this case the solution of the equation is $f=\sigma e^{u}$ and after the dilatation in $f$ we obtain $f=e^{u}$. Substituting this expression for $f$ into (3.4), we find the solution

$$
\tau=c_{5} t+c_{6}, \xi=c_{3} x+c_{4}, \eta=-\left(2 c_{3}+c_{5}\right) .
$$

Combining it with the above found solution for the function $f=e^{u}$, we obtain

$$
\tau=\tau(t), \xi=c_{3} x+c_{4}, \eta=-\left(\tau^{\prime}(t)+2 c_{3}\right) .
$$

1.5. If $c_{2} \neq 0$, by a shift in $u$ we can vanish $b$ and get the equation $u f^{\prime}(u)=\beta f(u)$, where $\beta=\frac{c_{2}-2 c_{3}-c_{5}}{c_{2}}$. A nonlinear solution as $2 c_{3}+c_{5} \neq 0,2 c_{3}+c_{5} \neq c_{2}$ is $f=\sigma u^{\beta}$ or $f=u^{\beta}$ after the dilatation w.r.t. $f$. At that, the linear cases $\beta=0, \beta=1$ are excluded from consideration. Substituting into (3.4), we obtain $b=0$ and the solution to this specification

$$
\tau=\left((1-\beta) c_{2}-2 c_{3}\right) t+c_{6}, \quad \xi=c_{3} x+c_{4}, \quad \eta=c_{2} u .
$$

It remains to study the case $c_{1} \neq 0$. Equation (3.8) can be represented as

$$
\frac{4 f^{\prime}(u)+u f^{\prime \prime}(u)}{f^{\prime \prime}(u)}=\frac{-b^{\prime}(x)}{c_{1}} .
$$

The left hand side is a function of $u$, the right hand side is a function of $x$. Thus, these functions are constant. Let $\frac{-b^{\prime}(x)}{c_{1}}=c_{7}$, then $b=-c_{1} c_{7} x+c_{8}$. After the shift in $u$ by $c_{7}$ we get the equation

$$
4 f^{\prime}(u)+f^{\prime \prime}(u) u=0 .
$$

Its solution $f=\sigma u^{-3}+\delta$ or after the dilatation $f=u^{-3}+\delta$. We substitute these functions $f$, $b$ into (3.4) and multiply by $u^{4}$. We obtain the equation

$$
u\left(c_{2}-2 c_{3}-\tau^{\prime}(t)\right)+3\left(c_{2} u-c_{1} c_{7} x+c_{8}\right)+\delta u^{4}\left(c_{2}-3 c_{1} x-2 c_{3}-\tau^{\prime}(t)\right)=0 .
$$

The case $\delta \neq 0$ does not give the expansion for the kernel of the algebras. Consider the case $\delta=0$. Splitting the latter equation in $x, u$, we obtain

$$
4 c_{2}-2 c_{3}-\tau^{\prime}(t)=0, c_{7}=c_{8}=0 .
$$

It shows that $\tau=\left(4 c_{2}-2 c_{3}\right) t+c_{6}, b=0$ and thus $\eta=\left(c_{1} x+c_{2}\right) u$.

1.6. Thus, for the function $f=u^{-3}$ we obtain the solution

$$
\tau=\left(4 c_{2}-2 c_{3}\right) t+c_{6}, \quad \xi=c_{1} x^{2}+c_{3} x+c_{4}, \quad \eta=\left(c_{1} x+c_{2}\right) u .
$$

This case expands the algebra obtained for an arbitrary power function. Thus, the case $\alpha=0$ is completely studied.

As $\alpha>0$, each equation can be reduced to an equivalent equation with $\alpha=1$, and as $\alpha<0$, to the equation $\alpha=-1$. 
We return back to equation (3.3) of the determining system assuming $\alpha=1$. Then employing identity $\xi_{x x}=2 \eta_{x u}$, we obtain

$$
\eta_{x x u}=\frac{\xi_{x x x}}{2},-\xi_{x x x}+4 \xi_{x}=0 .
$$

The general solution to the latter equation reads as

$$
\xi=c_{1} e^{2 x}+c_{2} e^{-2 x}+c_{3},
$$

then

Substituting it to equation (3.3), we get

$$
\eta=\left(c_{1} e^{2 x}-c_{2} e^{-2 x}+c_{4}\right) u+b(t, x) .
$$

$$
\begin{aligned}
b_{t}(t, x) & -b_{t x x}(t, x)+f(u)\left(c_{4}-3 c_{1} e^{2 x}+3 c_{2} e^{-2 x}-\tau^{\prime}(t)\right) \\
& -f^{\prime}(u)\left(\left(c_{1} e^{2 x}-c_{2} e^{-2 x}+c_{4}\right) u+b(t, x)\right)=0 .
\end{aligned}
$$

2.1. The case $f=0$ gives the equation $b_{t}(t, x)-b_{t x x}(t, x)=0$ that implies $b=\sigma(t) e^{x}+$ $\delta(t) e^{-x}+\gamma(x)$. Thus,

$$
\begin{aligned}
& \tau=\tau(t), \quad \xi=c_{1} e^{2 x}+c_{2} e^{-2 x}+c_{3}, \\
& \eta=\left(c_{1} e^{2 x}-c_{2} e^{-2 x}+c_{4}\right) u+\sigma(t) e^{x}+\delta(t) e^{-x}+\gamma(x) .
\end{aligned}
$$

2.2. As $f=1$, we obtain the equation

$$
b_{t}(t, x)-b_{t x x}(t, x)=3 c_{1} e^{2 x}-3 c_{2} e^{-2 x}-c_{4}+\tau^{\prime}(t) .
$$

This equation is integrated first for function $b_{t}$ and then we integrate it w.r.t. $t$. The general solution reads as

$$
\begin{aligned}
& b=d_{1}(t) e^{x}+d_{2}(t) e^{-x}+\left(-c_{1} e^{2 x}+c_{2} e^{-2 x}-c_{4}\right) t+\tau(t)+d_{3}(x), \\
& \tau=\tau(t), \quad \xi=c_{1} e^{2 x}+c_{2} e^{-2 x}+c_{3}, \\
& \eta=\left(c_{1} e^{2 x}-c_{2} e^{-2 x}+c_{4}\right)(u-t)+d_{1}(t) e^{x}+d_{2}(t) e^{-x}+\tau(t)+d_{3}(x) .
\end{aligned}
$$

2.3. Let $f=u$, then after the substitution into (3.10) we get

$$
b_{t}(t, x)-b_{t x x}(t, x)+u\left(-4 c_{1} e^{2 x}+4 c_{2} e^{-2 x}-\tau^{\prime}(t)\right)-b(t, x)=0,
$$

this is why $4 c_{1} e^{2 x}-4 c_{2} e^{-2 x}+\tau^{\prime}(t)=0, c_{1}=0, c_{2}=0, \tau=c_{5}$. We obtain the coefficients of the generators

$$
\tau=c_{5}, \quad \xi=c_{3}, \quad \eta=c_{4} u+b(t, x),
$$

where $b$ is the solution to equation

$$
b_{t}(t, x)-b_{t x x}(t, x)-b(t, x)=0 .
$$

Suppose now that $f$ is a nonlinear function. We differentiate equation (3.10) w.r.t. $u$

$$
f^{\prime}(u)\left(4 c_{1} e^{2 x}-4 c_{2} e^{-2 x}+\tau^{\prime}(t)\right)+f^{\prime \prime}(u)\left(\left(c_{1} e^{2 x}-c_{2} e^{-2 x}+c_{4}\right) u+b(t, x)\right)=0 .
$$

Differentiating equation (3.12) w.r.t. $t$, we obtain

$$
f^{\prime}(u) \tau^{\prime \prime}(t)+f^{\prime \prime}(u) b_{t}(t, x)=0 .
$$

If $b_{t}=0$, then $\tau=c_{5} t+c_{6}$. We consider one more differential consequence of equation (3.12) by differentiating it w.r.t. $x$ :

$$
\left(8 f^{\prime}(u)+2 u f^{\prime \prime}(u)\right)\left(c_{1} e^{2 x}+c_{2} e^{-2 x}\right)+f^{\prime \prime}(u) b^{\prime}(x)=0 .
$$

If $c_{1}=c_{2}=0$, then $b$ is a constant. Substituting the obtained expression into (3.10), we get

$$
f(u)\left(c_{4}-c_{5}\right)-f^{\prime}(u)\left(c_{4} u+b\right)=0 .
$$

As $c_{4}=c_{5}=0$ we have $b=0$ and there are no symmetries additional to the kernel. 
2.4. If $c_{4}=0$, applying the dilatation in $u$, we obtain equation $f^{\prime}(u)=f(u)$ with solution $f=e^{u}$ (after the dilatation in $f$ ). The coefficients of the generator read as

$$
\tau=c_{5} t+c_{6}, \quad \xi=c_{3}, \quad \eta=-c_{5} .
$$

2.5. If $c_{4} \neq 0$, by a shift in $u$ we can vanish $b$. The solution of the remaining equation after a dilatation in $f$ is the function $f=u^{\beta}$, where $\beta=\frac{c_{4}-c_{5}}{c_{4}}, \beta \neq 0, \beta \neq 1$. The coefficients of the symmetries operator cast into the form

$$
\tau=(1-\beta) c_{4} t+c_{6}, \quad \xi=c_{3}, \quad \eta=c_{4} u .
$$

The second case is $c_{1} \neq 0$ or $c_{2} \neq 0$. Then equation (3.14) becomes

$$
-\frac{8 f^{\prime}(u)+2 u f^{\prime \prime}(u)}{f^{\prime \prime}(u)}=\frac{b^{\prime}(x)}{c_{1} e^{2 x}+c_{2} e^{-2 x}}=\gamma, \quad c_{1}^{2}+c_{2}^{2} \neq 0, \gamma=\text { const. }
$$

After the shift in $u$, we arrive at the equation $4 f^{\prime}(u)+u f^{\prime \prime}(u)=0$ for the function $f$.

2.6. One can see that $f=u^{-3}+\delta$. We substitute this function into (3.10)

$$
\left(u^{-3}+\delta\right)\left(-3 c_{1} e^{2 x}+3 c_{2} e^{-2 x}+c_{4}-c_{5}\right)+3 u^{-4}\left(\left(c_{1} e^{2 x}-c_{2} e^{-2 x}+c_{4}\right) u+b\right)=0
$$

or

$$
u^{-3}\left(4 c_{4}-c_{5}\right)+\delta\left(-3 c_{1} e^{2 x}+3 c_{2} e^{-2 x}+c_{4}-c_{5}\right)+3 b u^{-4}=0 .
$$

The case $\delta \neq 0$ gives no additional symmetries. Suppose $\delta=0$. Then $b=0, c_{5}=4 c_{4}$. The solution to the determining equations is

$$
\tau=4 c_{4} t+c_{6}, \quad \xi=c_{1} e^{2 x}+c_{2} e^{-2 x}+c_{3}, \quad \eta=\left(c_{1} e^{2 x}-c_{2} e^{-2 x}+c_{4}\right) u .
$$

Suppose now $b_{t} \neq 0$, then equation (3.13) can be transformed to

$$
\frac{f^{\prime \prime}(u)}{f^{\prime}(u)}=-\frac{\tau^{\prime \prime}(t)}{b_{t}(t, x)}=\gamma=\text { const } \neq 0 .
$$

We can achieve $\gamma=1$ by the dilatation in $u$. Then $b(t, x)=-\tau^{\prime}(t)+\psi(x), f=\sigma e^{u}+\delta$. Employing a shift in $u$ and a dilatation in $f$, we can obtain $f=e^{u}+\delta$, where $\delta=0$ or $\delta= \pm 1$. We substitute these functions into (3.10) to obtain equation

$$
\begin{array}{r}
-\tau^{\prime \prime}(t)+\left(e^{u}+\delta\right)\left(-3 c_{1} e^{2 x}+3 c_{2} e^{-2 x}+c_{4}-\tau^{\prime}(t)\right) \\
-e^{u}\left(\left(c_{1} e^{2 x}-c_{2} e^{-2 x}+c_{4}\right) u-\tau^{\prime}(t)+\psi(x)\right)=0 .
\end{array}
$$

It yields $c_{1}=c_{2}=c_{4}=0, \psi(x)=0, \tau^{\prime \prime}(t)+\delta \tau^{\prime}(t)=0$. The case $\delta=0$ leads us to the same solution as in Item 2.4 .

2.7. Let $\delta \neq 0$, then $\tau=c_{5} e^{-\delta t}+c_{6}, b=c_{5} \delta e^{-\delta t}$. The coefficients of the operator for the case $f=e^{u}+\delta, \delta \neq 0$, become

$$
\tau=c_{5} e^{-\delta t}+c_{6}, \quad \xi=c_{3}, \quad \eta=c_{5} \delta e^{-\delta t} .
$$

It remains to study the case $\alpha=-1$. Arguing as for $\alpha=1$, we obtain the equation for $\xi$

$$
\xi_{x x x}+4 \xi_{x}=0 .
$$

Its solution is $\xi=c_{1} \cos 2 x+c_{2} \sin 2 x+c_{3}$, then $\eta_{x u}=\frac{1}{2} \xi_{x x}=-2 c_{1} \cos 2 x-2 c_{2} \sin 2 x$. It yields $\eta_{u}=-c_{1} \sin 2 x+c_{2} \cos 2 x+c_{4}$, since $\eta_{u u}=\eta_{t u}=0$. Thus, $\eta=\left(-c_{1} \sin 2 x+c_{2} \cos 2 x+\right.$ $\left.c_{4}\right) u+b(t, x)$. Proceeding as in the case $\alpha=1$, one can obtain similar specifications with the symmetries groups given in the table.

Substituting the obtained results into equation (3.3), we get

$$
\begin{aligned}
-b_{t}(t, x) & -b_{t x x}(t, x)+f(u)\left(3 c_{1} \sin 2 x-3 c_{2} \cos 2 x+c_{4}-\tau^{\prime}(t)\right) \\
& -f^{\prime}(u)\left(\left(-c_{1} \sin 2 x+c_{2} \cos 2 x+c_{4}\right) u+b(t, x)\right)=0 .
\end{aligned}
$$


3.1. As $f=0$, equation (3.15) becomes $b_{t}(t, x)+b_{t x x}(t, x)=0$, then $b=c_{5}(t) \sin x+$ $c_{6}(t) \cos x+c_{7}(x)$. The solution to the determining equations is

$$
\begin{aligned}
& \tau=\tau(t), \quad \xi=c_{1} \cos 2 x+c_{2} \sin 2 x+c_{3}, \\
& \eta=\left(-c_{1} \sin 2 x+c_{2} \cos 2 x+c_{4}\right) u+c_{5}(t) \sin x+c_{6}(t) \cos x+c_{7}(x) .
\end{aligned}
$$

3.2. Let $f=1$, we then obtain the equation

$$
\left.b_{t}(t, x)+b_{t x x}(t, x)=3 c_{1} \sin 2 x-3 c_{2} \cos 2 x+c_{4}-\tau^{\prime}(t)\right) .
$$

The general solution to this equation is

$$
b(t, x)=d(t) \sin x+e(t) \cos x+\left(-c_{1} \sin 2 x+c_{2} \cos 2 x+c_{4}\right) t-\tau(t)+h(x) .
$$

The coefficients of the generators then become

$$
\begin{gathered}
\tau=\tau(t), \quad \xi=c_{1} \cos 2 x+c_{2} \sin 2 x+c_{3}, \\
\eta=\left(-c_{1} \sin 2 x+c_{2} \cos 2 x+c_{4}\right)(u+t)+d(t) \sin x+e(t) \cos x-\tau(t)+h(x) .
\end{gathered}
$$

3.3. Let $f=u$. We substitute this function into (3.15), we obtain

$$
-b_{t}(t, x)-b_{t x x}(t, x)+u\left(4 c_{1} \sin 2 x-4 c_{2} \cos 2 x-\tau^{\prime}(t)\right)-b(t, x)=0 .
$$

Therefore, $c_{1}=c_{2}=0, \tau=c_{5}$. The coefficients of the generators read as

$$
\tau=c_{5}, \quad \xi=c_{3}, \quad \eta=c_{4} u+b(t, x),
$$

where $b$ solves the equation

$$
b_{t}(t, x)+b_{t x x}(t, x)+b(t, x)=0 .
$$

Consider the case of a nonlinear function $f$. Differentiating (3.15) w.r.t. $u$, we obtain

$$
\begin{aligned}
& f^{\prime}(u)\left(4 c_{1} \sin 2 x-4 c_{2} \cos 2 x-\tau^{\prime}(t)\right) \\
& -f^{\prime \prime}(u)\left(\left(c_{4}-c_{1} \sin 2 x+c_{2} \cos 2 x\right) u+b(t, x)\right)=0 .
\end{aligned}
$$

Differentiating (3.17) w.r.t. $t$, we obtain

$$
f^{\prime}(u) \tau^{\prime \prime}(t)+f^{\prime \prime}(u) b_{t}(t, x)=0 .
$$

If $b_{t}=0$, then $\tau=c_{5} t+c_{6}$. If we differentiate equation (3.17) w.r.t. $x$, we obtain a differential consequence

$$
\left(8 f^{\prime}(u)+2 u f^{\prime \prime}(u)\right)\left(c_{1} \cos 2 x+c_{2} \sin 2 x\right)-f^{\prime \prime}(u) b^{\prime}(x)=0 .
$$

Let $c_{1}=c_{2}=0$, then $b$ is a constant and equation (3.15) is written as

$$
f(u)\left(c_{4}-c_{5}\right)-f^{\prime}(u)\left(c_{4} u+b\right)=0 .
$$

3.4. If $c_{4}=0$, employing the dilatation w.r.t. $u$ and $f$, we obtain the solution $f=e^{u}$. Then $b=-c_{5}$ and the solution to the determining system of equations reads as

$$
\tau=c_{5} t+c_{6}, \quad \xi=c_{3}, \quad \eta=-c_{5} .
$$

3.5. In the case $c_{4} \neq 0$ we argue as for $\alpha=1$ to obtain the specification $f=u^{\beta}$, where $\beta=\frac{c_{4}-c_{5}}{c_{4}}, \beta \neq 0, \beta \neq 1$. The coefficients of the generator of the symmetries group read as

$$
\tau=c_{4}(1-\beta) t+c_{6}, \quad \xi=c_{3}, \quad \eta=c_{4} u .
$$

Suppose now $c_{1}^{2}+c_{2}^{2} \neq 0$. Then equation (3.19) can be written as

$$
\frac{8 f^{\prime}(u)+2 u f^{\prime \prime}(u)}{f^{\prime \prime}(u)}=\frac{b^{\prime}(x)}{c_{1} \cos 2 x+c_{2} \sin 2 x}=\gamma=\text { const. }
$$

After a shift in $u$, for function $f$ we obtain the equation $4 f^{\prime} u+u f^{\prime \prime}(u)=0$.

3.6. The latter equation implies $f=u^{-3}+\delta$. We substitute this function into (3.15) and get

$$
u^{-3}\left(4 c_{4}-c_{5}\right)+\delta\left(3 c_{1} \sin 2 x-3 c_{2} \cos 2 x+c_{4}-c_{5}\right)+3 b(x) u^{-4}=0 .
$$


The case $\delta \neq 0$ produces no additional symmetries. Let $\delta=0$. Then $b=0, c_{5}=4 c_{4}$. The solution to determining equation read as

$$
\tau=4 c_{4} t+c_{6}, \quad \xi=c_{1} \cos 2 x+c_{2} \sin 2 x+c_{3}, \quad \eta=\left(-c_{1} \sin 2 x+c_{2} \cos 2 x+c_{4}\right) u .
$$

Suppose now $b_{t} \neq 0$, then equation $(3.18)$ can be reduced to the form

$$
\frac{f^{\prime \prime}(u)}{f^{\prime}(u)}=-\frac{\tau^{\prime \prime}(t)}{b_{t}(t, x)}=\gamma=\mathrm{const} \neq 0
$$

One can obtain $\gamma=1$ employing the dilatation w.r.t. $u$. Then $b(t, x)=-\tau^{\prime}(t)+\psi(x), f=e^{u}+\delta$, $\delta=0$ or $\delta= \pm 1$. We substitute this function into (3.15):

$$
\begin{aligned}
\tau^{\prime \prime}(t) & +\left(e^{u}+\delta\right)\left(3 c_{1} \sin 2 x-3 c_{2} \cos 2 x+c_{4}-\tau^{\prime}(t)\right) \\
& -e^{u}\left(\left(-c_{1} \sin 2 x+c_{2} \cos 2 x+c_{4}\right) u-\tau^{\prime}(t)+\psi(x)\right)=0 .
\end{aligned}
$$

It follows that $c_{1}=c_{2}=c_{4}=0, \psi(x)=0, \tau^{\prime \prime}(t)-\delta \tau^{\prime}(t)=0$. The case $\delta=0$ is considered above.

3.7. Let $\delta \neq 0$, then the latter equation has the solution $\tau=c_{5} e^{\delta t}+c_{6}$ that yields $b=-c_{5} \delta e^{\delta t}$. The coefficients of the operator become

\begin{tabular}{|c|c|c|c|}
\hline & $\alpha=0$ & $\alpha=1$ & $\alpha=-1$ \\
\hline$f=0$ & $\begin{array}{l}\tau(t) \partial_{t}, \partial_{x}, x \partial_{x}, u \partial_{u} \\
x^{2} \partial_{x}+x u \partial_{u}, d(t) \partial_{u} \\
e(x) \partial_{u}, c(t) x \partial_{u}\end{array}$ & $\begin{array}{l}\tau(t) \partial_{t}, \partial_{x}, u \partial_{u}, \\
e^{2 x}\left(\partial_{x}+u \partial_{u}\right) \\
e^{-2 x}\left(\partial_{x}-u \partial_{u}\right) \\
\gamma(x) \partial_{u}, \sigma(t) e^{x} \partial_{u} \\
\delta(t) e^{-x} \partial_{u}\end{array}$ & $\begin{array}{l}\tau(t) \partial_{t}, \partial_{x}, u \partial_{u}, \\
\cos 2 x \partial_{x}-u \sin 2 x \partial_{u}, \\
\sin 2 x \partial_{x}+u \cos 2 x \partial_{u}, \\
c_{5}(t) \sin x \partial_{u}, \\
c_{6}(t) \cos x \partial_{u}, c_{7}(x) \partial_{u}\end{array}$ \\
\hline$f=1$ & $\begin{array}{l}2 \tau(t) \partial_{t}-\tau(t) x^{2} \partial_{u}, \\
\partial_{x}, x \partial_{x}-t x^{2} \partial_{u}, \\
2 x^{2} \partial_{x}+\left(2 x u-t x^{3}\right) \partial_{u}, \\
\left(2 u+t x^{2}\right) \partial_{u}, \quad d(t) \partial_{u}, \\
e(x) \partial_{u}, c(t) x \partial_{u}\end{array}$ & $\begin{array}{l}\tau(t)\left(\partial_{t}+\partial_{u}\right), \partial_{x} \\
e^{2 x}\left(\partial_{x}+(u-t) \partial_{u}\right) \\
e^{-2 x}\left(\partial_{x}-(u-t) \partial_{u}\right) \\
e^{x} d_{1}(t) \partial_{u} \\
e^{-x} d_{2}(t) \partial_{u} \\
d_{3}(x) \partial_{u}\end{array}$ & $\begin{array}{l}\tau(t)\left(\partial_{t}-\partial_{u}\right), \partial_{x} \\
\cos 2 x \partial_{x}-(u+ \\
+t) \sin 2 x \partial_{u} \\
\sin 2 x \partial_{x}+(u+ \\
+t) \cos 2 x \partial_{u} \\
d(t) \sin x \partial_{u} \\
e(t) \cos x \partial_{u}, h(x) \partial_{u}\end{array}$ \\
\hline$f=$ & $\begin{array}{l}\partial_{t}, \partial_{x}, u \partial_{u}, 2 t \partial_{t}-x \partial_{x} \\
b_{(3.5)}(t, x) \partial_{u}\end{array}$ & $\begin{array}{ll}\partial_{t}, & \partial_{x}, \\
b_{(3.11)}(t, x) \partial_{u} & \end{array}$ & $\begin{array}{ll}\partial_{t}, & \partial_{x}, \\
b_{(3.16)}(t, x) \partial_{u} & \end{array}$ \\
\hline$f=e^{u}$ & $\begin{array}{l}\tau(t) \partial_{t}-\tau^{\prime}(t) \partial_{u}, \partial_{x} \\
x \partial_{x}-2 \partial_{u}\end{array}$ & $\partial_{t}, \partial_{x}, t \partial_{t}-\partial_{u}$ & $\partial_{t}, \partial_{x}, t \partial_{t}-\partial_{u}$ \\
\hline$f=u^{\beta}$ & $\begin{array}{l}\partial_{t}, \partial_{x}, 2 t \partial_{t}-x \partial_{x} \\
(1-\beta) t \partial_{t}+u \partial_{u}\end{array}$ & $\begin{array}{l}\partial_{t}, \partial_{x}, \\
(1-\beta) t \partial_{t}+u \partial_{u}\end{array}$ & $\begin{array}{l}\partial_{t}, \partial_{x} \\
(1-\beta) t \partial_{t}+u \partial_{u}\end{array}$ \\
\hline$f=u^{-3}$ & $\begin{array}{l}\partial_{t}, \partial_{x}, 2 t \partial_{t}-x \partial_{x} \\
4 t \partial_{t}+u \partial_{u}, x^{2} \partial_{x}+x u \partial_{u}\end{array}$ & $\begin{array}{l}\partial_{t}, \quad \partial_{x}, \quad 4 t \partial_{t}+u \partial_{u} \\
e^{2 x}\left(\partial_{x}+u \partial_{u}\right) \\
e^{-2 x}\left(\partial_{x}-u \partial_{u}\right)\end{array}$ & $\begin{array}{l}\partial_{t}, \partial_{x}, 4 t \partial_{t}+u \partial_{u} \\
\cos 2 x \partial_{x}-u \sin 2 x \partial_{u} \\
\sin 2 x \partial_{x}+u \cos 2 x \partial_{u}\end{array}$ \\
\hline $\begin{array}{l}f=e^{u}+\delta \\
\delta= \pm 1\end{array}$ & & $\partial_{t}, \partial_{x}, e^{-\delta t}\left(\partial_{t}+\delta \partial_{u}\right)$ & $\partial_{t}, \partial_{x}, e^{\delta t}\left(\partial_{t}-\delta \partial_{u}\right)$ \\
\hline
\end{tabular}

$$
\tau=c_{5} e^{\delta t}+c_{6}, \quad \xi=c_{3}, \quad \eta=-c_{5} \delta e^{\delta t},
$$

where $f=e^{u}+\delta, \delta= \pm 1$.

Table 1 


\section{CONClusion}

The results of the group classification are summarized in Table 1 above. The function $b_{(i . j)}(t, x)$ solves equation with the number $(i . j)$. All the other functions are assumed to be arbitrary.

The results of the work can be used for the search of invariant and partially invariant solutions to the equation.

\section{BIBLIOGRAPHY}

1. H. Gajewski, K. Gröger, K. Zacharias. Nichtlineare Operatorgleichungen und Operatordifferentialgleichungen. Akademie-Verlag, Berlin. 1974.

2. A.G. Sveshnikov, A.B. Al'shin, M.O. Korpusov, Yu.D. Pletner. Linear and nonlinear equation of Sobolev type. Fizmatlit, Moscow, 2007. (in Russian.)

3. N.J. Hoff. Creep buckling // Aeron. Quarterly. 1956. V. 7, No. 1. P. 1-20.

4. L.V. Ovsyannikov. The "PODMODELI" program. Gas dynamics. // Prikl. Matem. Mekhanika. 1994. V. 58, No. 4. P. 29-55. [J. Appl. Math. Mech. 1994. V. 58, No. 4. P. 601-627.]

5. L.V. Ovsyannikov. Group analysis of differential equations. Nauka, Moscow, 1978. [Academic Press, New York, 1982.]

6. S.V. Meleshko. Group classification of the equations of two-dimensional motions of a gas // Prikl. Matem. Mekhanika. 1994. V. 58, No. 4. P. 56-62. [J. Appl. Math. Mech. 1994. V. 58, No. 4. P. 629635.]

7. S.V. Khabirov. Group classification of Hamilton systems // Dynamics of solid state. Novosibirsk. 1980. V. 44. P. 139-146. (in Russian.)

8. Yu.A. Chirkunov, S.V. Khabirov. Elements of symmetry analysis for equations of solid state mechanics. Novosibirsk State Technical University, Novosibirsk. 2012. (in Russian.)

Alexandr Vasil'evich Panov, Chelyabinsk State University,

Br. Kashirinych str., 129, 454001, Chelyabinsk, Russia

E-mail: gjd@bk.ru 